\title{
Multi-objective optimization of thermal comfort and energy consumption in a typical office room using CFD and NSM-PSO
}

\author{
$\underline{\text { Nan Li }}{ }^{\text {a }}$, Sherman C.P. Cheung ${ }^{\text {a }}$, Xiaodong Li ${ }^{b}$ and Jiyuan Tu ${ }^{\text {a }}$ \\ ${ }^{a}$ The School of Aerospace, Mechanical and Manufacturing Engineering, RMIT University, Australia \\ ${ }^{b}$ The School of Computer Science and IT, RMIT University, Australia \\ Email: s3468780@student.rmit.edu.au
}

\begin{abstract}
With ever increasing energy costs, finding an optimal design to strike a balance between thermal comfort, indoor air quality and energy consumption has become a constant challenge for engineers. A number of research works have been carried out to develop a computational design optimization method for the indoor environment where design indices such as predicted mean vote (PMV), percentage dissatisfied of draft (PD), age of air, $\mathrm{CO}_{2}$ concentration and energy cost were considered. Nevertheless, previous studies mainly focused on single objective optimization procedures where artificial weighting factors were introduced to combine all design objectives into one single objective function. It is well known that the optimal design may be sensitive to the weighting factors. Different weighting factors could result in substantially different optimal solutions.

In attempting to resolve the aforementioned problem, this paper provides some preliminary results on the development of a multi-objective optimization algorithm, which could be integrated into generic computational fluid dynamic (CFD) packages. A nondominated sorting-based multi-objective particle swarm optimization (NSM-PSO) algorithm has been developed to perform design optimization for a typical office room based on CFD predictions. The supply air temperature and velocity are the design parameters selected to optimize against the predicted mean vote (PMV), $\mathrm{CO}_{2}$ concentration and energy consumption as objective functions. The results show that the optimal design temperature ranges from $290.15 \mathrm{~K}$ to $294.15 \mathrm{~K}$, and the velocity ranges between $0.15 \mathrm{~m} / \mathrm{s}$ and $0.44 \mathrm{~m} / \mathrm{s}$ where a $3 \mathrm{D}$ Pareto-optimal front is given within the range. Based on the given Pareto-optimal front, designers could then choose the optimal design which is wellbalanced between thermal comfort, air quality and energy consumption.
\end{abstract}

Keywords: Multi-objective optimization, computational fluid dynamics (CFD), nondominated particle swarm optimization (PSO) 


\section{INTRODUCTION}

In recent decades, with the development of computational simulation technology, the usage of numerical methods in engineering design has attracted significant attention in the literature. Compared to conventional design cycle, numerical simulations offer a faster and more economical way for engineers to assess or predict the design performance and its relationship to different design parameters. It is envisioned that the numerical approaches will become important tools in engineering design and optimization in the near future. Computational Fluid Dynamics (CFD) has aroused much attention from heating, ventilation and airconditioning (HVAC) system researchers. Many studies have been performed to assess and optimize the performance of a given HVAC system using CFD techniques. "Fong et al. (2006)" proposed a novel simulation-EP (i.e. evolutionary programming) coupling approach to optimize the energy consumption for the HVAC system in a local subway station. "Lin et al. (2009)" studied the effect of partitions in an office on the performance of under floor air supply ventilation system via CFD simulation. "Zhou and Haghighat (2009a, b)" published their research results about ventilation system optimization using the CFD-ANN (Artificial Neural Network)-GA (Genetic Algorithm) combination method in 2009. "Stavrakakis et al. (2011)" did some research on the window size selection for optimizing occupational comfort. "Li et al. (2013)" proposed a POD (Proper orthogonal decomposition) model in their research of ventilation system operation optimization. "Zhai et al. (2014)" developed an inverse design method for indoor ventilation system using CFD and GA.

In order to quantify the system performance, some common design indices such as predicted mean vote (PMV), percentage dissatisfied of draft (PD), age of air, $\mathrm{CO}_{2}$ concentration and energy cost are considered as optimization objective in literature. In most of the previous works, the multiple design indices were blended to a single objective problem using artificial aggregating or weighting factors. A generic form of objective function constructed by aggregating and weighting several indices is given by:

$$
f(\mathrm{x})=\omega_{1} f_{1}(\mathrm{x})+\omega_{2} f_{2}(\mathrm{x})+\cdots+\omega_{n} f_{n}(\mathrm{x})
$$

where $\omega_{1}, \ldots, \omega_{n}$ are the aggregating/weighting factors. One obvious drawback of this method is that the optimal design could be critically sensitive to the weighting factors. In other words, different sets of weighting factors could result in substantially different optimal solutions. Therefore, these weighting factors must be chosen very carefully in the optimization procedure. In addition, this method of handling a multiobjective optimization gives only one optimal solution, which means the designers have no flexibility in selecting alternative solutions. As an attempt to overcome the aforementioned shortcoming, in this study, we propose the use of a nondominated sorting-based multi-objective particle swarm optimization (NSM-PSO) algorithm to achieve multi-objective optimization without any weighting factors. This population-based algorithm, as an improved technique of the basic particle swarm optimization (PSO), can give a group of nondominated (i.e. non-biased) solutions, providing the engineers with multiple options from which they can select the most appropriate design based on professional judgment or end-user input "Carrese et al. (2011)".

In essence, the HVAC system design is a multi-objective optimization problem where the objectives are generally conflictive with each other. For example, we prefer a cooler office environment in hot summer. Meanwhile, we would also like to reduce the energy consumption of air-conditioning. Nonetheless, in general, the energy consumption is inversely proportion to the supply air temperature. Therefore, the optimization algorithm aims at seeking a trade-off between several different design objectives. The vectorvalued of multi-objective optimization is of the form:

$$
f(\mathrm{x})=\left(f_{1}(\mathrm{x}), f_{2}(\mathrm{x}), \cdots, f_{n}(\mathrm{x})\right)
$$

where the vector $f$ represents $n$ conflicting objectives. The theoretical solutions of multi-objective optimization problem are called Pareto Front, which is constituted by the whole nondominated solutions. The traditional solution from weighting method is only one point located on the Pareto Front. The NSM-PSO is capable of finding a host of points which are well distributed on the Pareto Front, and providing designers more flexibility in choosing their favorite solutions.

In this paper, we present some preliminary results on the development of a multi-objective optimization algorithm which could be integrated into generic CFD packages. The two state-of-the-art numerical techniques (CFD and NSM-PSO) are combined together to assess and optimize the performance of a HVAC system design in a typical office room. In the following preliminary study, among all design parameters of the system, the temperature and the velocity of the supply air (i.e. the two critical design variables) were selected for assessment and optimization. The system performance will be evaluated against thermal comfort, 
air quality and energy consumption. The predicted mean vote (PMV), $\mathrm{CO}_{2}$ concentration and energy consumption are therefore selected to construct the objective functions.

\section{METHODOLOGY}

In order to perform a multi-objective optimization, a sufficient data set of input-output that represents the response of the system performance in relation to each design parameter, should be generated in advance. As mentioned above, a generic CFD framework has been adopted as a reliable predictive tool to construct the input-output data space. To ensure the validity of the simulation, predictions of the CFD model were validated against full-scale experimental data by Yuan et al. (1999). After validation, simulations with different control variables (i.e. inlet temperature and velocity) were performed to obtain the corresponding system performance for the output space (i.e. PMV, $\mathrm{CO}_{2}$ concentration, energy consumption). The output space was then passed into the NSM-PSO to perform an iterative optimization process which searches the Pareto front (i.e. finds optimal trade-off solutions). Multidimensional interpolation is applied to calculate the fitness value of particles. A schematic of the overall methodology is depicted in Figure 1.

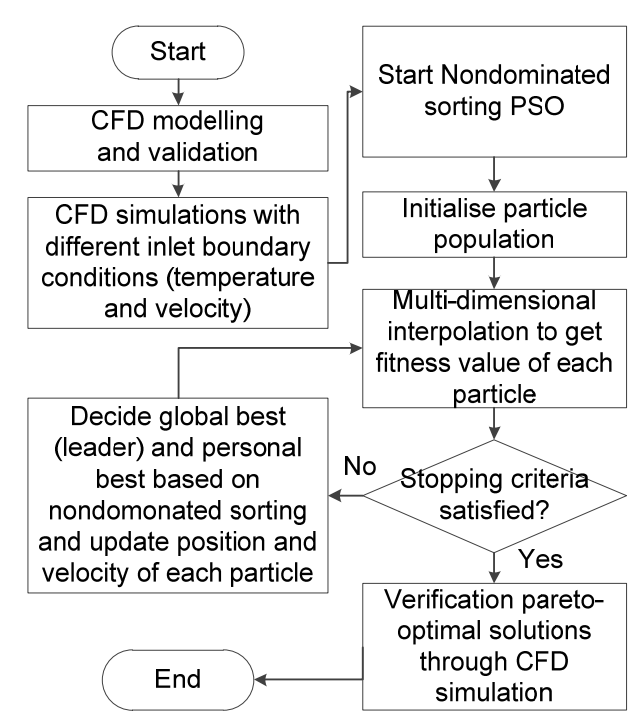

Figure 1. CFD-based multi-objective optimization system framework.

\subsection{Basic Particle Swarm Optimization}

The particle swarm optimization (PSO) has been widely adopted as a population-based stochastic optimization method. The method was first introduced by "Kennedy (2001)" based on the inspiration drawn from observing social behaviors of ants which include learning from previous experience and communicating with successful individuals. In the PSO algorithm, each particle has its own position and velocity, which are represented by $x_{i}$ and $v_{i}$, respectively and they are updated according to the following equations:

$$
\begin{aligned}
& v_{i}(\mathrm{t}+1)=\omega v_{i}(\mathrm{t})+c_{1} \varphi_{1}\left(p_{i}-x_{i}(\mathrm{t})\right)+c_{2} \varphi_{2}\left(p_{g}-x_{i}(\mathrm{t})\right) \\
& x_{i}(\mathrm{t}+1)=x_{i}(\mathrm{t})+v_{i}(\mathrm{t}+1)
\end{aligned}
$$

where $p_{i}$ and $p_{g}$ represent the personal best position and global best position, respectively, and $c_{1}$ and $c_{2}$ are two uniform random numbers within the range $[0,1]$. The $\varphi_{1}$ and $\varphi_{2}$ are two constants which are set to 2 . The parameter $\omega$ decreases with increasing iteration number within the range [0.4, 1.2]. To avoid overflow of the search space, both the position and velocity are limited within boundaries, $\left[x_{\min }, x_{\max }\right]$ and $\left[v_{\min }, v_{\max }\right]$, respectively. Unfortunately, the original architecture of the PSO is only capable to solve a single-objective optimization problem.

\subsection{Nondominated Sorting-based Multi-objective PSO}

In order to enable the basic PSO to solve multi-objective optimization problem, "Li (2003)" applied a Nondominated Sorting Method (NSM) to the original PSO inspired by "Deb et al. (2002)". In NSM-PSO, the updating equations for particle position and velocity do not change, but the selection methods of personal best and global best are different. Nondominated comparison between particles' personal bests and their offspring is used to decide the new personal bests. Nondominated sorting is carried out in a temporary population which consists of $N$ particles' personal bests and $N$ their offspring (therefore $2 N$ individuals) to decide the nondomination rank of each individual. Then the global best is selected from the group which has top nondomination rank and in order to avoid local optimal aggregation, crowding distance is calculated and sorted. Therefore, the global best must meet both the following requirements: top nondomination rank and largest crowding distance. Throughout the iteration process, the particles are moving towards the Paretooptimal Front guided by the leader (global best) and are well distributed because of population diversity maintenance (crowding distance). 


\section{OPTIMIZATION OBJECTIVES}

In the present study, the thermal comfort, air quality and energy consumption were taken into consideration for the assessment of the HVAC system performance. In contrast to most previous research, we optimize the three objectives simultaneously in the optimization process, rather than integrating all of them into one objective function with weighting factors. The definition of the Predicted Mean Vote, $\mathrm{CO}_{2}$ concentration, and energy consumption are briefly discussed as follows.

\subsection{Predicted Mean Vote}

The predicted mean vote (PMV) is a thermal comfort evaluation index which was first introduced by "Fanger (1972)". This value represents the mean subjective satisfaction with the indoor thermal environment with a number between -3 (cold) and +3 (hot). Zero is defined as the ideal value representing thermal neutrality and our objective is to make $|\mathrm{PMV}|$ as small as possible. Fanger's equations are used to calculate the PMV with a particular combination of air temperature, mean radiant temperature, relative humidity, air speed, metabolic rate, and clothing insulation "Fanger (1972)". In this paper, we evaluated the average PMV based on the predicted field information obtained from CFD simulations.

\section{2. $\mathrm{CO}_{2}$ Concentration}

To assess the air quality within the space, the concentration of $\mathrm{CO}_{2}$ emitted by occupants throughout the office room was also resolved in the CFD simulation. In the simulation, the $\mathrm{CO}_{2}$ is emitted from the occupants with the velocity and concentration of $0.018 \mathrm{~m} \mathrm{~s}^{-1}$ and $4.0 \mathrm{ppm}$ respectively. Similar to the average $\mathrm{PMV}$, the average $\mathrm{CO}_{2}$ concentration was extracted from the predicted CFD field information.

\subsection{Energy Consumption}

The energy costs of air-conditioning can be divided into two parts: ventilation fan power and cooling/heating energy consumption "Li et al. (2013)". These two parts and total energy costs can be determined by the following equations:

$$
\begin{aligned}
& E_{\text {fan }}=\frac{P \cdot V_{\text {air }}}{\eta_{\text {fan }}} \\
& E_{\text {cooling/ heating }}=m_{\text {supply }} c_{p}\left(T_{\text {return }}-T_{\text {supply }}\right)+m_{\text {outdoor }}\left(h_{\text {outdoor }}-h_{\text {return }}\right) \\
& E_{\text {total }}=E_{\text {fan }}+E_{\text {cooling heating }}
\end{aligned}
$$

where $P$ is air pressure difference of the fan and $V$ is volume flow rate of supply air $\left(\mathrm{m}^{3} \mathrm{~s}^{-1}\right), m$ represents the mass flow rate of the air $\left(\mathrm{kg} \mathrm{s}^{-1}\right), c_{p}$ is the specific heat capacity of air, $T$ represents temperature, $h$ is the specific enthalpy of air $\left(\mathrm{J} \mathrm{kg}^{-1}\right)$ which is related to air temperature and relative humidity. Similarly, we can get energy costs from the CFD-Post package.

\section{SIMULATION RESULTS}

\subsection{Case Description}

In order to study the aforementioned HVAC system design optimization, a three-dimensional computational domain representing a typical office room was constructed. Figure 2 shows the geometry layout of the computational domain. The cooling air flows from the air-conditioning at the right and leaves the room from

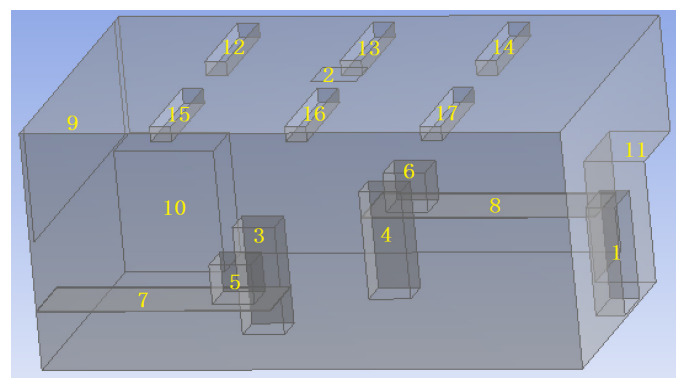

Figure 2. The layout of the typical office

Table 1. The boundary conditions.

\begin{tabular}{llll}
\hline Number & Name & Boundary details & Comments \\
\hline $\mathbf{1}$ & Air- & Normal speed \& & Controlled \\
$\mathbf{2}$ & conditioning & Static temperature & variables \\
$\mathbf{3 , 4}$ & Exhaust & Average static pressure & $0[\mathrm{~Pa}]$ \\
$\mathbf{5 , 6}$ & Occupant & Temperature & $37[\mathrm{C}]$ \\
$\mathbf{7 , 8}$ & Desktop & Heat flux & $108.5\left[\mathrm{~W} / \mathrm{m}^{2}\right]$ \\
$\mathbf{9}$ & Table & Adiabatic & ------ \\
$\mathbf{1 0 , 1 1}$ & Partition & Heat transfer coefficient & $3.7\left[\mathrm{~W} /\left(\mathrm{m}^{2} \mathrm{~K}\right)\right]$ \\
$\mathbf{1 2 - 1 7}$ & Furniture & Adiabatic & ------- \\
& Light & Heat flux & $34\left[\mathrm{~W} / \mathrm{m}^{2}\right]$ \\
& Room wall & Heat transfer coefficient & $0.19\left[\mathrm{~W} /\left(\mathrm{m}^{2} \mathrm{~K}\right)\right]$ \\
\hline
\end{tabular}


the exhaust at the center of the roof. Details of the boundary conditions are listed in table 1 . The temperature and velocity of the inlet are controlled variables and other boundary conditions are fixed for all simulations. To get enough sample data, a total of 25 simulations with different combinations of controlled variables were carried out; where the inlet temperature was chosen from $\{17,18,19,20,21\}\left[{ }^{\circ} \mathrm{C}\right]$ and the velocity was chosen from $\{0.1,0.2,0.3,0.4,0.5\}\left[\mathrm{m} \mathrm{s}^{-1}\right]$. In this study, the commercial CFD package - ANSYS CFX 14.5 was adopted to simulate air flow and heat transfer within the typical office room.

\subsection{CFD Simulation Results}

To ensure the validity of the CFD simulation, predictions of the CFD model were first validated against the full-scale experimental data reported by "Yuan et al. (1999)". Figure 3 shows the comparisons between the measured and predicted air temperature and velocity along a vertical line at the center of the office room under the inlet condition $\left(17^{\circ} \mathrm{C}, 0.09 \mathrm{~m} \mathrm{~s}^{-}\right.$ $\left.{ }^{1}\right)$. The blue lines are the results extracted from the
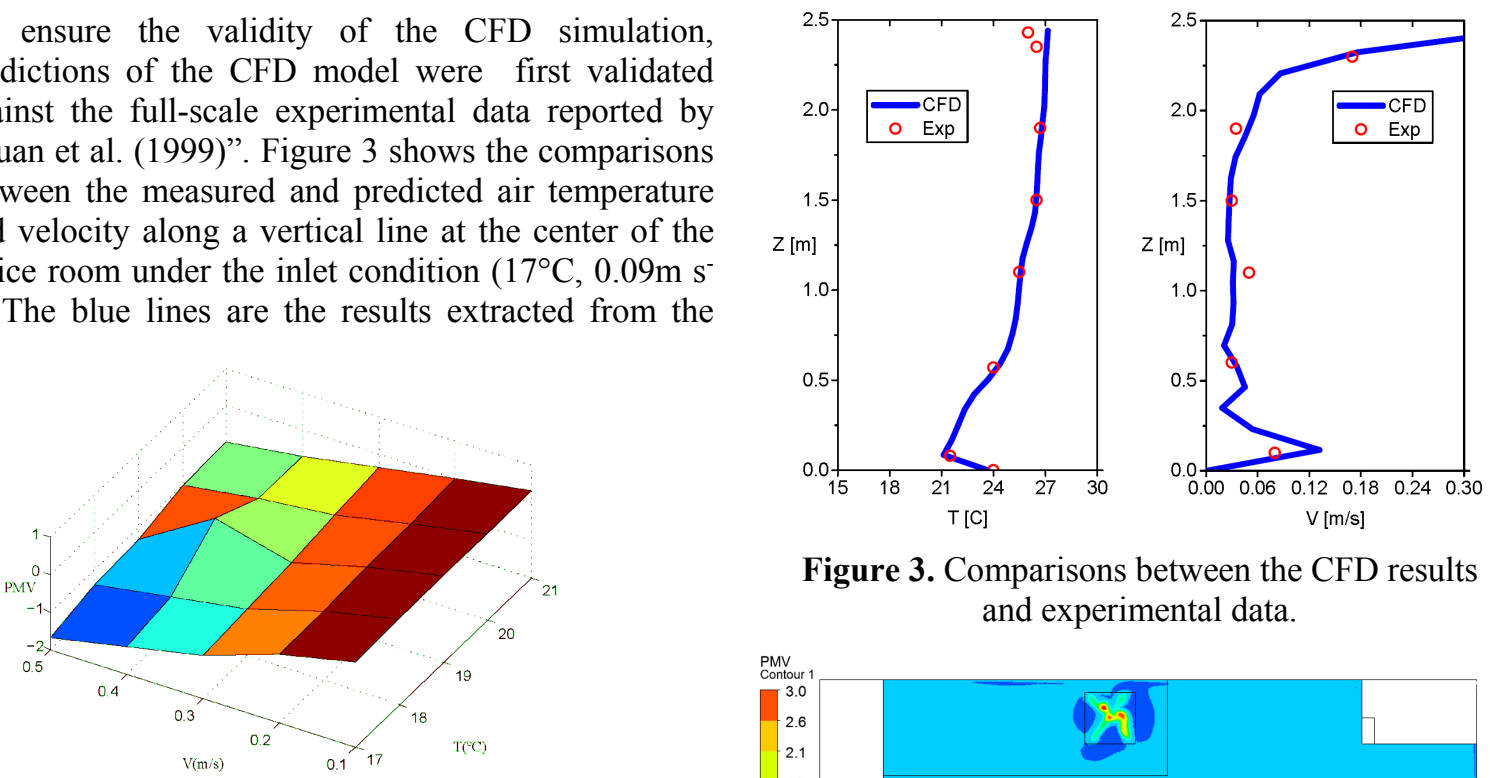

Figure 3. Comparisons between the CFD results and experimental data.
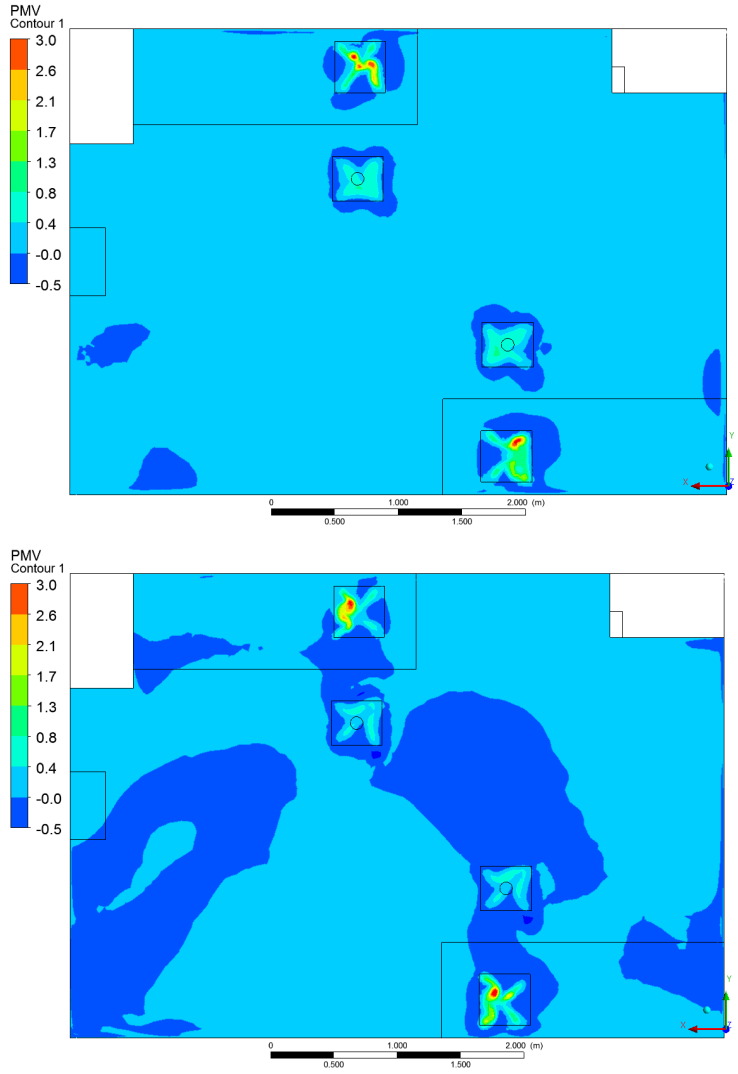

Figure 4. Response surfaces of the three objectives (PMV, energy, CO2).

Figure 5. Comparisons of PMV contour between baseline case (upper) and case 2 (lower).

CFD simulation and the red dots are the experimental data report by "Yuan et al. (1999)". As depicted, the predicted temperature and velocity are in satisfactory agreement with the experimental measurements; showing that the CFD predictions are reliable for design optimization. 
Afterwards, the validated CFD model was then applied to predict the response surface of the system performance (i.e. PMV, $\mathrm{CO}_{2}$ and energy) with respect to different design parameters (i.e. supply air temperature and velocity). The corresponding response surface is shown in Figure 4. The response surface was then passed to the NSM-PSO for multi-objective optimization. The optimization results are discussed in next section.

\subsection{Optimization Results}

In order to make a comparison, two sets of optimization methods were performed in the present study. The first method adopts a similar approach as in previous works using weighting factors to construct a single objective function. The second method adopts the proposed NSM-PSO to find multiple equally good and well-distributed solutions.

\section{Weighting method}

In this optimization process, two objectives (i.e. PMV and energy consumption) are selected to construct the objective function, given by:

$$
f=\omega_{1} f_{P M V}+\omega_{2} f_{\text {Energy }}
$$

where $\omega_{1}$ and $\omega_{2}$ are the weighting parameters which determine the optimization results. Table 2 shows the impact of weighting factors on the optimal results and Figure 5 shows a comparison of PMV contour between the Baseline case $\left(17^{\circ} \mathrm{C}, 0.1\right.$ $\left.\mathrm{m} \mathrm{s}^{-1}\right)$ and the improved Case $2\left(20.6^{\circ} \mathrm{C}, 0.17\right.$ $\mathrm{m} \mathrm{s}^{-1}$ ).

For Case 1 in Table 2, it can be observed that both the thermal comfort and energy consumption are improved in comparison to the baseline case (i.e. $33 \%$ and $23 \%$, respectively). Comparing Case 1 with Case 2, by reducing the weighing factor for energy, a higher (i.e. $81 \%$ ) thermal comfort improvement could be achieved. Nonetheless, the Case 3 would cost $10 \%$ more energy compared with the baseline case. Therefore, we can conclude that the results are sensitive to weighting factors and this method can only output one solution in each run. Moreover, because different designers would have different preferences, it is difficult to fix the weighting factors in advance and only giving one solution per run provides no flexibility in choosing alternative trade-off solutions.

\section{Nondominated sorting method}

To remedy the drawback of the first method, the NSM-PSO is a weighting factor free optimization procedure, and multiple trade-off solutions can be provided in one run. In this paper, the iteration number is set to be 100 and the nondominated solutions by NSM-PSO method are shown in Figures 6-9, where each of the blue dots represents a solution in the objective space. Figures 6-8 indicate the nondominated solutions of 2-objective problems provided by the NSM-PSO (PMV-Energy, $\mathrm{CO}_{2}$-Energy and $\mathrm{CO}_{2}$-PMV, respectively), and Figure 9 shows the nondominated solutions of a 3-objective problem considering all the three objectives (PMV-Energy- $\mathrm{CO}_{2}$ ). Obviously, the NSM-PSO provides multiple nondominated solutions (i.e. improvement in terms of one objective comes from a sacrifice on at least one of other objectives), providing designers with

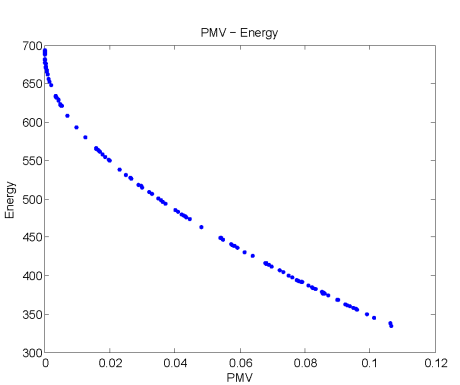

Figure 6. Nondominated solutions considering two objectives: PMV and energy.

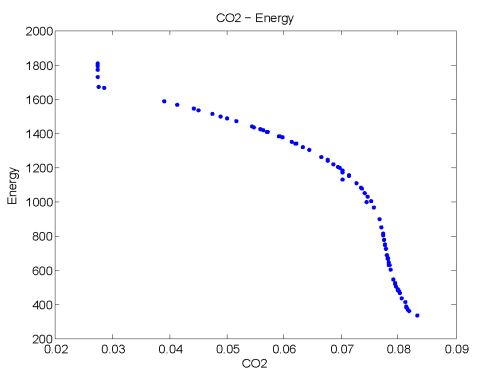

Figure 7. Nondominated solutions considering two objectives: $\mathrm{CO}_{2}$ concentration and energy.

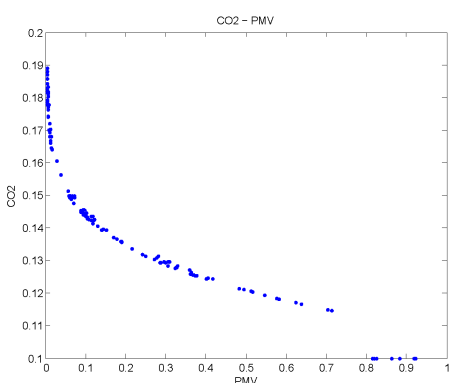

Figure 8. Nondominated solutions considering two objectives: $\mathrm{CO}_{2}$ concentration and PMV. 
flexibility in choosing alternative solutions which are equally good. After getting the optimal Pareto front, engineers can select one set of design parameter from the front according to their professional judgments or end-user preferences.

\section{CONCLUSIONS}

This paper presents some preliminary results on the development of a multi-objective optimization algorithm which is tailored to be integrated with generic CFD packages. The commercial CFD package - ANSYS CFX was adopted to simulate and predict the air flow and heat transfer in a typical office room. A total of 25 CFD simulations with different combinations of inlet temperature and velocity were carried out to obtain the response surface for optimization. Different with previous research, the NSM-PSO was utilized to perform multi-objective optimization, which is capable to find multiple different but equally good optimal solutions with only one optimization run. The results show that the combination of CFD and NSM-PSO is a feasible and promising method for multi-objective engineering optimization design.

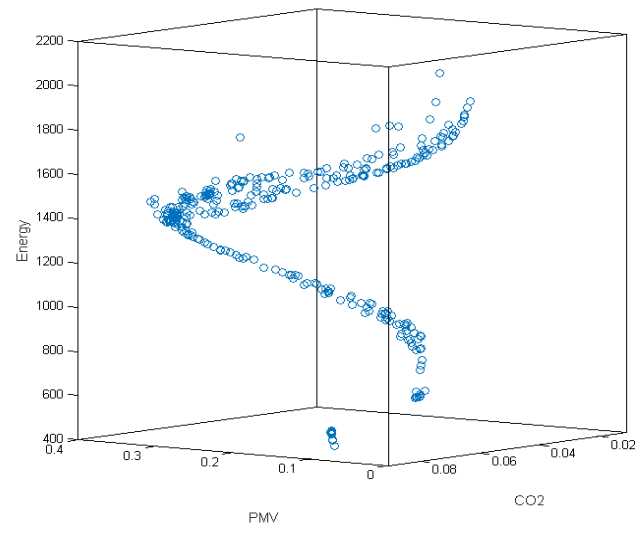

Figure 9. Nondominated solutions considering three objectives: $\mathrm{PMV}, \mathrm{CO}_{2}$ concentration and energy.

\section{ACKNOWLEDGMENTS}

The financial support provided by an Australian Research Council grant (ARC Linkage LP130100236) is gratefully acknowledged.

\section{REFERENCES}

Carrese, R., A. Sobester, H. Winarto, and X. Li. (2011). "Swarm heuristic for identifying preferred solutions in surrogate-based multi-objective engineering design." AIAA Journal.

Deb, K., A. Pratap, S. Agarwal, and T. Meyarivan. (2002). "A fast and elitist multiobjective genetic algorithm: NSGA-II." Evolutionary Computation, IEEE Transactions on 6 (2):182-197. doi: $10.1109 / 4235.996017$.

Fanger, P. O. (1972). Thermal comfort: analysis and applications in environmental engineering. New York: McGraw-Hill.

Fong, K. F., V. I. Hanby, and T. T. Chow. (2006). "HVAC system optimization for energy management by evolutionary programming." Energy and Buildings 38 (3):220-231.

Kennedy, James. (2001). Swarm intelligence. Edited by Russell C. Eberhart and Yuhui Shi. San Francisco: San Francisco: Morgan Kaufmann Publishers.

Li, K., W. Xue, C. Xu, and H. Su. (2013). "Optimization of ventilation system operation in office environment using POD model reduction and genetic algorithm." Energy and Buildings 67:34-43.

Li, X. (2003). A non-dominated sorting particle swarm optimizer for multiobjective optimization. Springer.

Lin, Zhang, T. T. Chow, C. F. Tsang, K. F. Fong, L. S. Chan, W. S. Shum, and Luther Tsai. (2009). "Effect of internal partitions on the performance of under floor air supply ventilation in a typical office environment." Building and Environment 44 (3):534-545. doi: 10.1016/j.buildenv.2008.04.018.

Stavrakakis, G. M., D. P. Karadimou, P. L. Zervas, H. Sarimveis, and N. C. Markatos. (2011). "Selection of window sizes for optimizing occupational comfort and hygiene based on computational fluid dynamics and neural networks." Building and Environment 46 (2):298-314.

Yuan, Xiaoxiong, Qingyan Chen, Leon R. Glicksman, Yongqing Hu, and Xudong Yang. (1999). "Measurements and computations of room airflow with displacement ventilation." ASHRAE Transactions 105:PART $1 \%$

Zhai, Zhiqiang, Yu Xue, and Qingyan Chen. (2014). "Inverse design methods for indoor ventilation systems using CFD-based multi-objective genetic algorithm." An International Journal 7 (6):661-669. doi: 10.1007/s12273-014-0179-2.

Zhou, L., and F. Haghighat. (2009a). "Optimization of ventilation system design and operation in office environment, Part I: Methodology." Building and Environment 44 (4):651-656.

Zhou, L., and F. Haghighat. (2009b). "Optimization of ventilation systems in office environment, Part II: Results and discussions." Building and Environment 44 (4):657-665. 